Eurostudia

\title{
Vorwort des wissenschaftlichen Konferenzbeirates
}

\section{Yuichi Morii, Barbara Thériault et Michael Werner}

Volume 7, numéro 1-2, 2011

Deutschland und Europa : Grenzen und Grenzgänge(r)

L'Allemagne et l'Europe : frontières et passeurs

URI : https://id.erudit.org/iderudit/1015006ar

DOI : https://doi.org/10.7202/1015006ar

Aller au sommaire du numéro

Éditeur(s)

Le Centre canadien d'études allemandes et européennes

ISSN

1718-8946 (numérique)

Découvrir la revue

Citer ce document

Morii, Y., Thériault, B. \& Werner, M. (2011). Vorwort des wissenschaftlichen Konferenzbeirates. Eurostudia, 7(1-2), vii-ix. https://doi.org/10.7202/1015006ar

Tous droits réservés @ Le Centre canadien d'études allemandes et européennes, 2012
Ce document est protégé par la loi sur le droit d'auteur. L'utilisation des services d'Érudit (y compris la reproduction) est assujettie à sa politique d'utilisation que vous pouvez consulter en ligne.

https://apropos.erudit.org/fr/usagers/politique-dutilisation/ 


\title{
VORWORT DES WISSENSCHAFTLICHEN KONFERENZBEIRATES
}

\author{
Yuichi Morii \\ Zentrum für Deutschland- und Europastudien, Universität Tokyo (DESK), Komaba \\ Barbara Thériault \\ Centre canadien d'études allemandes et européennes (CCEAE), Université de \\ Montréal \\ Michael Werner \\ Centre interdisciplinaire d'études et de recherches sur l'Allemagne (CIERA) / EHESS, \\ Paris
}

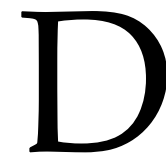

ie Beiträge des vorliegenden Bandes gehen aus der Konferenz „Deutschland und Europa: Grenzen und Grenzgänge(r)“ hervor, die der Deutsche Akademische Austauschdienst (DAAD) in Kooperation mit dem Wissenschaftszentrum Berlin für Sozialforschung (WZB) im Mai 2010 in Berlin veranstaltete.

Die Konferenz stellt ein Novum dar: Erstmals tauschten sich die Forscher an den zwanzig interdisziplinären Zentren für Deutschland- und Europastudien, die der DAAD seit 1991 in elf Ländern auf drei Kontinenten initiierte und fördert(e), über ein Querschnittsthema der Deutschland- und Europastudien fachlich aus. Angesprochen wurden mit der Ausschreibung der "Grenzen- und Grenzgänge(r) “-Konferenz (Nachwuchs-)Wissenschaftlerinnen und Wissenschaftler an den DAAD-Zentren in den USA, Kanada, Großbritannien, Frankreich, den Niederlanden, Polen, Bulgarien, der Russischen Föderation, China, Japan und Israel, darüber hinaus aber auch weitere Kreise in- und ausländischer Deutschlandexpertinnen und -experten aus unterschiedlichen Disziplinen der Geistes-, Kultur- und Sozialwissenschaften, um den vielstimmigen und - wie sich in Berlin zeigte: höchst lebendigen - Dialog über dieses Kernthema aktueller Deutschland- und Europastudien auch in diesem Sinne „über die Grenzen hinweg“ zu führen. Ein zentrales Anliegen der Konferenz war es also, bezogen auf die Leitthematik, in und mit diesem Teilnehmer- und Referentenkreis eine Kombination der Blicke von außen und von innen auf Deutschland und Europa herzustellen und die in den vom DAAD geförderten Zentren gesammelten Ressourcen in einen fruchtbaren Austausch mit deutschen und internationalen Wissenschaftlerinnen und Wissenschaftlern $\mathrm{zu}$ bringen. Die Zusammenarbeit mit dem WZB als einer der führenden interdisziplinären und internationalen Forschungseinrichtungen in Deutschland bildete dabei eine ideale Partnerschaft. Es erschien uns zudem besonders wichtig, junge Forscherinnen und 
Forscher aktiv in das Berliner Forum einzubeziehen: Die Impulse, die wir aus den Interessen und Zugängen der Nachwuchswissenschaftlerinnen und Nachwuchswissenschaftler beziehen können, sind für die Produktivität und die kontinuierliche Weiterentwicklung unserer academic community unverzichtbar; umgekehrt ist der Austausch für junge Wissenschaftlerinnen und Wissenschaftler im internationalen Umfeld der Deutschland- und Europastudien ein wesentlicher Schritt in die akademische Zukunft. Gleichzeitig spiegelte sich in der Begegnung erfahrener und junger Forscherinnen und Forscher auf der Berliner Konferenz ein verbindendes Element aller DAAD-Zentren wider: Sie bündeln in ihren jeweiligen Ländern nicht nur die wissenschaftliche Beschäftigung mit Deutschland und Europa und vermitteln diese oft auch an eine breitere Öffentlichkeit, sie alle engagieren sich darüber hinaus mit innovativen, interdisziplinären Master- und Doktoratsstudiengängen für die Ausbildung einer jungen Expertengeneration.

Die von uns für die Konferenz formulierte Leitthematik „Grenzen und Grenzgänge(r)“ zielte darauf, neue Forschungen in diesem in den vergangenen zwei Jahrzehnten viel bearbeiteten und für Deutschland und Europa besonders relevanten Themenfeld zur Diskussion zu stellen und verschiedene disziplinäre Zugangsweisen zu verknüpfen. Die Diskussion um Grenzziehung, Grenzräume, Überwindung von und Umgang mit Grenzen wurde und wird in den einzelnen Fächern der Sozial- und Kulturwissenschaften unterschiedlich geführt. Die wissenschaftlichen Disziplinen haben verschiedene Perspektiven entwickelt, die bislang nur unzureichend miteinander kommunizieren. Die gegenseitige Kenntnis umeinander und den Austausch über unterschiedliche Zugänge zu fördern, bildete daher ein weiteres Hauptanliegen der Konferenz, von dem die hier publizierten, ausgewählten Beiträge ein beredtes Zeugnis ablegen. Mit den Stichworten „Grenzgänge“ und "Grenzgänger" rückten sowohl das gesellschaftliche, politische und kulturelle Handeln an und mit Grenzen als auch dessen Akteure in den Mittelpunkt des Interesses.

Die Diskussion des übergreifenden Konferenzthemas wurde in Berlin in sieben Themenfelder aufgefächert, die keinen disziplinspezifischen Vorgaben folgten, wenngleich manche das eine oder andere Fach mehr angesprochen haben mögen. Im Themenfeld "Grenzen und Grenzräume" konzentrierte sich die Debatte auf binneneuropäische Grenzräume als Aktionszusammenhänge und die Auswirkungen grenzübergreifender Kontakte auf Identitäts- und Gruppenbildungsprozesse sowie auf die Hinterfragung des politischen Umgangs mit Grenzräumen (siehe die Beiträge von Leslie/Clunan und Opiłowska). Darüber hinaus wurde die Leitthematik auf das dynamische Konzept der „kulturellen Räume“ bezogen, das die Vorstellung geschlossener Kulturräume ablöst, und insbesondere in Hinblick auf Berlin seit 1989 untersucht wurde (siehe die Beiträge von Thijs und Sheffi). Einen weiteren Schwerpunkt bildete die Beschäftigung mit "literarischen Grenzgänge(r)n" in Deutschland und Europa, deren Hintergründe, Entwicklungen und Auswirkungen untersucht wurden (siehe die Beiträge von Colvin, Ma, Simo und Wolff). Grenze und 
Grenzgänger bilden darüber hinaus klassische Gegenstände der Migrationsforschung, einem anderen Themenschwerpunkt der Konferenz, bei dem Eigenheiten im Verhältnis von Minderheiten und Mehrheiten in Deutschland und Europa herausgearbeitet wurden (siehe den Beitrag von Akiyama). Die damit verbundenen unterschiedlichen Aspekte "transnationaler Identitäten " wurden ebenfalls in den Blick genommen (siehe die Beiträge von Jürgens und v. Mering), etwa - aber nicht nur - mit der Frage, welche Effekte nationale Integrationspolitiken auf die Entstehung oder Abwesenheit "transnationaler Identitäten“ in modernen europäischen Gesellschaften zeitigen (siehe den Beitrag von Williams). Der Blick auf die "Grenzen Europas" erlaubte die Auseinandersetzung u.a. mit den neuen Außengrenzen der EU (siehe den Beitrag von Tomaszewska). Beiträge zur "Transformation von Staatlichkeit" untersuchten Natur und Ausmaß der Veränderungen, die der Nationalstaat im Europa der letzten Jahrzehnte erfahren hat, und beleuchteten Perspektiven für das 21. Jahrhundert (siehe die Beiträge von Escach und Wissel).

Wir freuen uns, dass es gelungen ist, eine repräsentative Auswahl der Konferenzbeiträge im vorliegenden Sonderheft von Eurostudia einem breiteren Publikum zugänglich zu machen. Wir sind sicher, dass sich daraus zahlreiche Anregungen für die weitere, interdisziplinäre Beschäftigung mit Deutschland und Europa ergeben. 